\title{
Sudden and unexpected deaths in an adult population, Cape Town, South Africa, 2001-2005
}

\author{
Marianne Tiemensma, Elsie Helena Burger
}

Background. According to the regulations of the National Health Act, all 'sudden and unexpected' deaths in South Africa should be referred to Forensic Pathology Services (FPS) for further investigation.

Objectives. We aimed to determine the final outcomes of forensic postmortem examinations in 'sudden and unexpected' adult deaths referred to Tygerberg FPS between 2001 and 2005.

Methods. The study was a retrospective descriptive study. Demographic and autopsy data from adult cases of 'sudden and unexpected deaths' referred to Tygerberg FPS were collected and analysed.

Results. Some 816 adult cases of 'sudden' deaths were studied. The presumed manner of death was natural in $645(79.0 \%)$ cases, unnatural in $99(12.2 \%)$, and undetermined in $72(8.8 \%)$. Diseases of the cardiovascular, respiratory and central nervous systems accounted for the majority of natural deaths. Infectious diseases accounted for most deaths in the youngest age group studied (18 29 years); however, ischaemic heart disease was the most prevalent cause of death in the total study population.

Conclusions. This study highlighted the lack of useful preautopsy information and therefore the performance of 'unnecessary' medicolegal autopsies in a resource-limited country. Clinicians could ease the burden by giving useful information when referring cases to FPS. Feedback should be given to family members, especially where the cause of death may have an impact on surviving family members.

S Afr Med J 2012;102:90-94

\section{Definitions ${ }^{1}$}

Cause of death: The disease, condition or injury that leads directly or indirectly, immediately or subsequently to death.

Manner of death: Refers to the circumstances of how the cause of death arose. The manner of death may be natural, accidental, homicidal, suicidal, iatrogenic or undetermined.

Mechanism of death: Refers to the physiological derangement or biochemical disturbance produced by the cause of death (e.g. hypovolaemia due to a stab wound).

A main function of a forensic pathologist is to assist the court(s) in distinguishing between 'natural' and 'unnatural' deaths. For medicolegal investigations of death (defined in the Regulations of the National Health Act (Act 61 of 2003)), the following are deemed to be deaths due to unnatural causes:

(a) Any death due to physical or chemical influence, direct or indirect, and/or related complications.

(b) Any death, including those deaths which would normally be considered to be a death due to natural causes, which in the opinion of the medical practitioner, has been the result of an act of commission or omission which may be criminal in nature.

(c) Where the death is sudden and unexpected, or unexplained or where the cause of death is not apparent.

Furthermore, procedure-related deaths, as contemplated in section 56 of the Health Professions Act (Act 56 of 1974) (amended according to section 48 of the Health Professions Amendment Act (Act 29 of 2007)) also need to be investigated. The death of a person undergoing, or as a result of, a procedure of a therapeutic, diagnostic

Division of Forensic Medicine, Department of Pathology, Stellenbosch University, Tygerberg, W Cape

Marianne Tiemensma, MB ChB, Dip For Med (SA), FC For Path (SA), MMed For Path

Elsie Helena Burger, MB ChB, FC For Path (SA) MMed For Path or palliative nature, or of which any aspect of such a procedure has been a contributory cause, shall not be deemed to be a death from natural causes as contemplated in the Inquest Act (Act 58 of 1959), or the Births, Marriages and Deaths Registration Act (Act 51 of 1992).

Autopsies are vital to uncover unexpected unnatural deaths in cases of 'sudden and unexpected' deaths, e.g. deaths due to poisoning. There are various definitions of 'sudden and unexpected death'. The World Health Organization defines it as 'Death within 24 hours from the onset of symptoms, ${ }^{2}$ while Mason defines it as 'Unexpected death following so rapidly from the onset of symptoms that the cause of death could not be certified with confidence by a medical practitioner familiar with the patient.'

Little is published on the subgroup of cases referred to the Forensic Pathology Services (FPS) in South Africa as 'sudden and unexpected' adult deaths (SUDA). We aimed to: review the outcomes of these cases to provide knowledge which may help future planning of standardised operating procedures (SOPs); determine the demographics of SUDA cases; and assess the quality of pre-autopsy information available to the pathologist.

\section{Material and methods}

This was an observational, retrospective, descriptive study that included all adult cases reported to Tygerberg FPS as cases of 'sudden and unexpected' deaths from 1 January 2001 to 31 December 2005. An 'adult' was defined as any person 18 years and older.

'Sudden and unexpected' adult deaths were not specifically defined, but all cases referred to Tygerberg FPS as supposed 'sudden and unexpected' deaths were included. Similarly, strict time limit for existence of symptoms prior to death was not defined.

Copies of postmortem reports, contemporaneous notes, hospital records and collateral information filed in the archive at the Division of Forensic Medicine, Tygerberg, were retrieved and studied. Data were collected and entered using Epidata $3.1^{4}$ and Microsoft Excel. Information received prior to autopsy, by a forensic pathology officer interviewing a relative or friend, with concomitant completion of a questionnaire, was also reviewed. A forensic pathology officer is an employee of FPS who assists medical personnel with collection and identification of bodies, interviews with relatives, and other mortuary 
functions. The questionnaire covers basic questions on the deceased person's medical, occupational and social history, medication taken, and circumstances surrounding the death.

Data analysis was performed by the authors. All data were treated confidentially. Ethics approval was obtained from the Health Research Ethics Committee (HREC) of the Health Science Faculty, University of Stellenbosch (Ref. No. N09/09/256).

\section{Results}

A total of 13536 possible unnatural death cases were referred to Tygerberg FPS over the studied period, of which 826 cases were reported as being 'sudden and unexpected' adult deaths; 10 cases in which the final postmortem results were unavailable at the time were excluded. Therefore the total study population comprised 816 cases.

Autopsies were performed in $601(74.4 \%)$ of the 816 cases; the remainder (215/816) were handled as 'view and grant' cases, where a probable natural cause of death could be determined after reviewing the case history and performing an external examination alone.

Table I presents a summary of the demographic findings. The ethnic distribution of our study population approximates that of the total population in the subcouncils served by Tygerberg FPS. ${ }^{5}$ Death occurred at home in $68.0 \%$ of cases.

In the $601 / 816$ cases in which autopsies had been performed, the most common underlying cause of death was ischaemic heart disease, and diseases of the cardiovascular, respiratory and central nervous systems accounted for the majority of natural deaths (Table II).

In females, the most common cause of natural deaths was pneumonia, followed by ischaemic heart disease and pulmonary thrombo-embolism (Fig. 1). The three most common causes of natural deaths in males were ischaemic heart disease, pulmonary tuberculosis (TB) and pneumonia (Fig. 2).
Table I. Summary of results

\begin{tabular}{ll}
\hline Gender $(N(\%))$ & $561(69.0)$ \\
Male & $255(31.0)$ \\
Female & \\
Age (yrs) & $18-89$ years \\
Range & 42.6 years \\
Mean & 41 years \\
Median & 35 years \\
Mode & \\
Ethnic distribution $(N(\%))$ & $397(49.0)$ \\
Coloured & $327(40.0)$ \\
Black & $89(11.0)$ \\
White & $3(0.3)$ \\
Indian/Asian & \\
Place of death $(N(\%))$ & $555(68.0)$ \\
Home & $126(15.4)$ \\
Public place & $83(10.2)$ \\
Hospital & $21(2.6)$ \\
Motor vehicle & $17(2.1)$ \\
Work place & $12(1.5)$ \\
Jail & $6(0.7)$ \\
Sports ground & \\
Presumed manner of death $(N(\%))$ & $645(79.0)$ \\
Natural & $74(9.1)$ \\
Accidental & $15(1.8)$ \\
Homicidal & $10(1.2)$ \\
Suicidal & $72(8.8)$ \\
Undetermined &
\end{tabular}

\begin{tabular}{|c|c|c|}
\hline System (total No. of cases per system) & Most common causes of death & No. of cases (\% per system) \\
\hline \multirow[t]{3}{*}{ Cardiovascular $(\mathrm{N}=140)$} & Ischaemic heart disease ${ }^{*}$ & $106(75.7)$ \\
\hline & Infectious heart diseases $^{\dagger}$ & $12(8.6)$ \\
\hline & Ruptured aorta aneurysm & $8(5.7)$ \\
\hline \multirow[t]{3}{*}{ Respiratory $(\mathrm{N}=122)$} & Pneumonia & $45(36.9)$ \\
\hline & Pulmonary tuberculosis & $37(30.3)$ \\
\hline & Pulmonary thrombo-embolism & $21(17.2)$ \\
\hline \multirow[t]{4}{*}{ Central nervous system $(\mathrm{N}=61)$} & Infectious diseases $\ddagger$ & $18(29.5)$ \\
\hline & Spontaneous intracerebral haemorrhage & $12(19.7)$ \\
\hline & Ruptured saccular aneurysm & $12(19.7)$ \\
\hline & Epilepsy & $12(19.7)$ \\
\hline \multirow[t]{3}{*}{ Gastro-intestinal $(\mathrm{N}=24)$} & Peptic ulcer disease & $5(20.8)$ \\
\hline & Pancreatitis & $5(20.8)$ \\
\hline & Liver disease $\$$ & $4(16.7)$ \\
\hline \multirow[t]{3}{*}{ Genito-urinary $(\mathrm{N}=10)$} & Kidney disease & $4(40)$ \\
\hline & Pregnancy-related causes $\|$ & $3(30)$ \\
\hline & Malignancy & $2(20)$ \\
\hline \multicolumn{3}{|c|}{ *Includes acute myocardial ischaemia, acute myocardial infarction, chronic myocardial ischaemia, coronary artery disease. } \\
\hline \multicolumn{3}{|c|}{${ }^{\dagger}$ Includes infectious pericarditis, myocarditis and endocarditis. } \\
\hline \multicolumn{3}{|l|}{${ }^{*}$ Includes meningitis and brain abscess. } \\
\hline \multicolumn{3}{|c|}{${ }^{5}$ Includes fatty disease of the liver, liver cirrhosis, portal hypertension due to liver disease, undetermined liver disease. } \\
\hline \multirow{2}{*}{\multicolumn{3}{|c|}{ 'Includes pyelonephritis, glomerulonephritis. }} \\
\hline & & \\
\hline
\end{tabular}




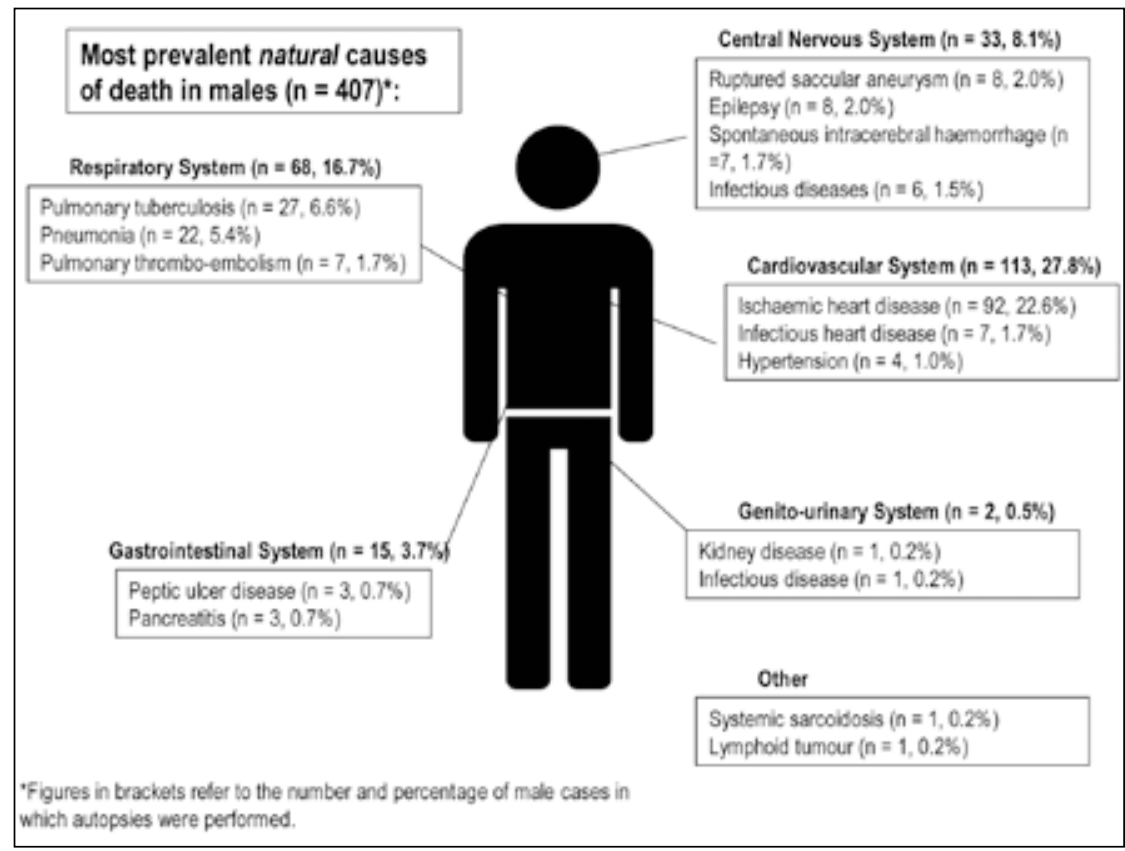

Fig. 1. Most prevalent causes of death in females.

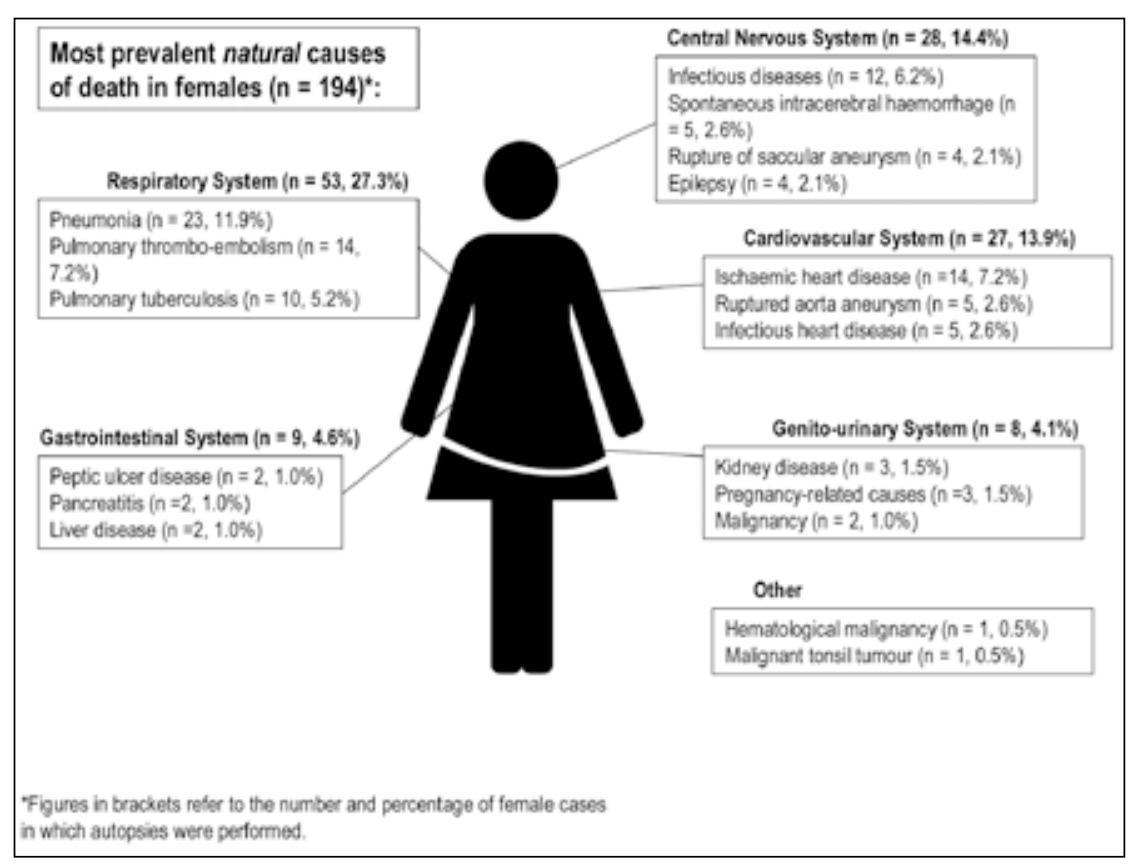

Fig. 2. Most prevalent causes of death in males.

Infectious diseases (pneumonia, meningitis) were the most common causes of death in the youngest age group (18 - 29 years). In all the other age groups, ischaemic heart disease was the most prevalent natural cause of death. Pulmonary TB was prevalent in all age categories (Table III).

In $99 / 601$ cases ( $16.5 \%$ of cases where autopsies were done) the manner of death was found to be unnatural. Death was attributed to acute alcohol poisoning in $35(5.8 \%)$ of these cases with an average blood alcohol concentration level of $0.38 \mathrm{~g} / 100$ $\mathrm{ml}$ (range $0.25-0.62 \mathrm{~g} / 100 \mathrm{ml}$ ). Death in 8 others was determined to have been caused by drugs or substances. These substances included tricyclic antidepressants, anticholinergic medication, anticonvulsant medication, morphine and MDMA ('Ecstasy', 4-methylenedioxymethamphetamine).
The percentage of cases in which the cause of death was 'undetermined with postmortem examination alone' was $10.6 \%$ (64/601), while the manner of death was undetermined in $12.0 \%(72 / 601)$ of cases where autopsies were done.

Information received prior to autopsy by a forensic pathology officer interviewing a relative or friend of the deceased was reviewed. Information was received in $83.1 \%$ $(678 / 816)$ of all cases, and in $81.4 \%(489 / 601)$ where autopsies had been performed.

After reviewing the history received from the informant and the completed autopsy report in each case, we tried to establish whether the supplied information contributed to the formulation of a cause of death (i.e. whether history and the cause of death could be correlated). This comparison was only done where full autopsies had been performed. Information was found to be most appropriate, and therefore contributed to the formulation of a cause of death, when supplied by a doctor $(p=0.001)$ (Fig. 3 ).

The most common symptoms reported were chest pain (51 cases) and abdominal pain (46 cases). The most common known medical disease reported was hypertension (73 cases) and TB (37 cases). The eventual causes of death in cases where chest pain was reported to be present before death were ischaemic heart disease, ruptured aortic aneurysm and pulmonary thromboembolism. Accordingly, 'chest pain' was the symptom which was most often in keeping with expected symptoms of the eventual cause of death (90.2\% - 46/51 cases).

Correlation between a known medical history prior to death, and the cause of death determined after the performance of an autopsy was evaluated. A history of 'ischaemic heart disease' correlated well with the cause of death determined at autopsy ( $2 / 2$ cases). A history of asthma showed no correlation with the eventual determined cause of death (4/4 cases), while epilepsy, $\mathrm{TB}$ and hypertension showed ambiguous results.

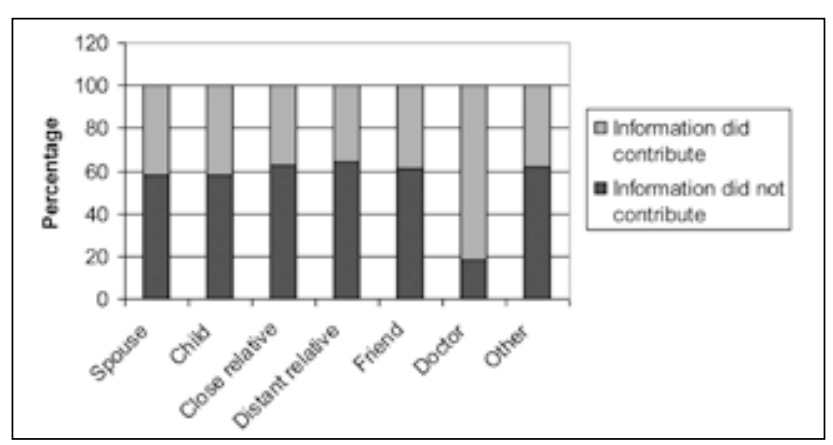

Fig. 3. Contribution of information by informants. 


\begin{tabular}{|c|c|c|}
\hline Age group (total No. of cases per age group) & Most prevalent natural causes of death* & No. of cases (\% of total per age group) \\
\hline \multirow{5}{*}{$18-29$ years $(N=88)$} & Pneumonia & $12(13.6)$ \\
\hline & Meningitis & $11(12.5)$ \\
\hline & Ischaemic heart disease & $6(6.8)$ \\
\hline & Infectious cardiovascular diseases & $6(6.8)$ \\
\hline & Pulmonary tuberculosis & $4(4.5)$ \\
\hline \multirow[t]{7}{*}{$30-39$ years $(N=115)$} & Ischaemic heart disease & $30(26.1)$ \\
\hline & Pulmonary tuberculosis & $17(14.8)$ \\
\hline & Pulmonary thrombo-embolism & $10(8.7)$ \\
\hline & Pneumonia & $7(6.1)$ \\
\hline & Ruptured saccular aneurysm & $4(3.5)$ \\
\hline & Epilepsy & $4(3.5)$ \\
\hline & Meningitis & $4(3.5)$ \\
\hline \multirow[t]{5}{*}{$40-49$ years $(N=100)$} & Ischaemic heart disease & $28(28)$ \\
\hline & Pulmonary tuberculosis & $11(11)$ \\
\hline & Spontaneous intracerebral haemorrhage & $6(6)$ \\
\hline & Epilepsy & $5(6)$ \\
\hline & Meningitis & $5(6)$ \\
\hline \multirow[t]{3}{*}{$50-59$ years $(N=68)$} & Ischaemic heart disease & $26(38.0)$ \\
\hline & Pneumonia & $6(8.8)$ \\
\hline & Pulmonary thrombo-embolism & $4(5.8)$ \\
\hline \multirow[t]{2}{*}{$60-69$ years $(N=22)$} & Ischaemic heart disease & $4(18.2)$ \\
\hline & Pulmonary thrombo-embolism & $2(9.1)$ \\
\hline \multirow[t]{2}{*}{ Older than 70 years $(N=18)$} & Ischaemic heart disease & $7(38.9)$ \\
\hline & Pulmonary tuberculosis & $2(11.1)$ \\
\hline
\end{tabular}

\section{Discussion \\ Outcomes of cases}

The most common organ systems involved in natural deaths (cardiovascular, respiratory and central nervous systems) and the most common cause of death (ischaemic heart disease) were in keeping with SUDA studies in France, ${ }^{6}$ Jamaica ${ }^{7}$ and South Carolina. ${ }^{8}$ Despite diverse settings, the similar outcomes are expected, as diseases or conditions of these organ systems are most likely to be responsible for 'true' SUDA cases, e.g. sudden cardiac death associated with cardiac dysrhythmia secondary to myocardial ischaemia.

Infectious diseases were the most prevalent natural cause of death in the youngest age group studied (18 - 29 years). Routine testing for HIV infection is not performed at our facility because of ethical considerations; therefore the impact of associated HIV infection on the underlying causes of death in these cases is unknown. However, it is suspected to be high, since many infections may present as opportunistic infections; TB is a good example, with a total of 37 deaths ascribed to TB. The impact of HIV disease on deaths in South Africa is seriously underestimated by official statistics, because of under-reporting. ${ }^{9}$ Ideally HIV testing of indicated cases should be performed, with appropriate management of the outcome of the results, as this may influence surviving family members or partners.

The Western Cape had the highest notification rate of smear-positive TB cases in South Africa in 2002. ${ }^{10}$ However, as TB is a chronic disease with a range of symptoms and signs, the large number of pulmonary TB cases found in this study was not anticipated. This finding may be explained by a lack of knowledge about the deceased's medical history, rather than the 'suddenness' of the death. Also, some cases arrived at FPS because the deceased did not have a 'regular' medical attendant who was willing to sign the death notification form.
Infectious diseases of the central nervous system as a cause of death (18/61 cases, $29.5 \%$ of central nervous system causes) were more common in this study than in other, international studies. A study on sudden unexplained death in adults due to intracranial pathology found the principal intracranial causes of death to be epilepsy (60.1\%), spontaneous subarachnoid haemorrhage by ruptured saccular aneurysm (18.8\%), and intracranial haemorrhage $(18.8 \%)$, with meningitis accounting for only $1 \% \cdot{ }^{11}$ The possibility of associated HIV infection in our cases is not known.

In South Africa, the manner of death is not determined by the forensic pathologist, but by the court(s), although the forensic pathologist has an important role in distinguishing between natural and unnatural deaths (see Table I for presumed manner of deaths). The presumed manner of death being unnatural in 99 cases (12.2\%) shows the importance of medicolegal autopsies, where a convincing and clear history of natural disease is not available, to uncover 'hidden/unexpected' unnatural causes of death.

Autopsies where no obvious cause of death could be found (negative autopsies) formed $10.6 \%(64 / 601)$ of cases, which is slightly higher than international results, e.g. $2.6 \%(44 / 1691)$ in the UK National Confidential Enquiry into Patient Outcome and Death study. ${ }^{2}$

\section{Alcohol poisoning}

The role of alcohol in traumatic deaths is frequently reported on; however, little has been published on its role in non-traumatic deaths. Death was attributed to acute alcohol poisoning in almost $6 \%(35 / 601)$ of our cases. This contrasts strongly with a medicolegal series of sudden adult death cases over a 5 -year study period in France, where alcohol was 'detected' in only 2 out of 77 cases $^{6}-$ the exact values of the blood alcohol concentration in these two cases 
were not supplied. Alcohol is the most frequently abused substance in the Western Cape, with the prevalence of lifetime alcohol use across household surveys ranging from $39 \%$ to $64 \%{ }^{12}$

\section{Information received prior to autopsy}

If no case history is available prior to autopsy, subtle pathology may be missed that may have been evident at autopsy if it was expected. It could also result in failure to perform or request special investigations, which may later prove to be important. Information is especially essential in alleged SUDA cases, to ascertain whether the death was indeed 'sudden and unexpected'. An effort should be made to reach family members and the general practitioner and to obtain clinical notes. Clinicians who refer cases to FPS should send detailed referral notes and their contact details. As the presumed manner of death in most of our cases was natural, it could be argued that many of the autopsies were superfluous, and necessitated only by the lack of useful pre-autopsy information.

Factors causing the high frequency of suboptimal information received before autopsy include: relatives may have been too distressed to talk, the deceased may have been unidentified when found, the general practitioner may not have been identified, or the police officer may have sought information that was irrelevant to the pathologist's requirements. ${ }^{13}$ Further reasons in South Africa include: language barriers, lack of medical training of forensic pathology officers who are the main interviewers of relatives or friends of the deceased, and poor access to transport for family members to attend an interview at the medicolegal facility.

Unfortunately, this study had a limited number of cases available for correlation between the symptoms and medical history, and the cause of death. Regarding distinguishing specific disease symptoms, many studies have shown that the verbal autopsy has different sensitivities and specificities when comparing different diagnoses. Further studies are needed to establish which symptoms and diseases are commonly found in our setting, and may be indicative of natural causes of death, and then a standard verbal autopsy questionnaire ${ }^{14}$ for the South African context should be developed.

\section{Feedback system}

It has been stressed ${ }^{15,16}$ that feedback regarding the cause of death should be given to relatives, especially where the cause of death could impact on the health of the surviving relatives and close contacts. The prevalence of ischaemic heart disease, pulmonary TB and infectious diseases of the central nervous system was high in this study. These are conditions where feedback could initiate preventive health measures that could save relatives' lives.

\section{Recommendations and conclusions}

In this study the majority of cases had a presumed natural manner and cause of death. We recommend the following to improve the quality and quantity of information received prior to autopsy, and possibly to reduce the number of unnecessary autopsies:

- Clinicians should try to obtain the medical history in SUDA cases and only refer these to FPS if no reasonable conclusion regarding a possible natural cause of death can be made, or if other factors necessitating a medicolegal autopsy exist.

- Clinicians should provide forensic pathologists with as much clinical history as possible.

- A Standard Verbal Autopsy Questionnaire should be developed for the South African context, suited to local needs, and preferably be available in local languages.

- Availability of translators to overcome language barriers.

\section{References}

1. Dada MA, McQuoid-Mason DJ. Introduction to Medico-legal Practice. Durban: Butterworths, 2001. 2. Saukko P, Knight B. Knight's Forensic Pathology. 3rd ed. London: Hodder Arnold, 2004

3. Mason JK. Forensic Medicine for Lawyers. 3rd ed. London: Butterworths, 1995 .
4. Lauritsen JM, Bruus M. EpiData (version 3). A comprehensive tool for validated entry and documentation of data. The EpiData Association, Odense Denmark, 2003-2004.

.

5. Cape Town Statistics from Census 2001. www.capetown.gov.za/statistics (accessed 1 November 2010). 6. De la Grandmaison G, Durigon M. Sudden adult death: a medico-legal series of 77 cases between 1995 and 2000. Med Sci Law 2002;42:225-232.

7. Escoffery CT, Shirley SE. Causes of sudden natural death in Jamaica: a medicolegal (coroner's) autopsy study from the University Hospital of the West Indies. Forensic Sci Int 2002;129:116-121.

8. Christiansen LR, Collins KA. Natural death in the forensic setting. A study and approach to the autopsy. Am J Forensic Med Pathol 2007;28:20-23.

9udkin PL, Burger EH, Bradshaw D, et al. Deaths caused by HIV disease under-reported in South Africa. AIDS 2009;23:1600-1602.

10. National Tuberculosis Control Program South Africa. Mobilizing against Tuberculosis. South African Plan for TB Control for 2002 to 2005. Pretoria: The Program, 2002.

11. Black M, Graham DI. Sudden unexplained death in adults caused by intracranial pathology. J Clin Pathol 2002;55:44-50.

12. Harker N, Kader R, Myers B, et al. Substance abuse trends in the Western Cape: a review of studies conducted since 2000 (Commissioned by the Department of Social Development, Cape Town). www. sahealthinfo.org/admodule/substance.pdf (accessed 1 February 2011).

13. Sampson H, Johnson A, Carter N, et al. Information before coronial necropsy: how much should be available? J Clin Pathol 1999;52:856-859.

14. World Health Organization. Verbal Autopsy Standards. Ascertaining and Attributing Cause of Death. Geneva: WHO, 2007.

15. Bowker TJ, Wood DA, Davies MJ, et al. Sudden, unexpected cardiac or unexplained death in England: a national survey. QJM 2003;96:266-279.

6. Lee A, Ackerman MJ. Sudden unexplained death: evaluation of those left behind. Lancet 2003;362:1429-1431.

Accepted 11 November 2011

\section{ERRATUM}

An error occurred in the South African Hypertension Guideline 2011 that was published as a supplement to January 2012 SAMJ. The error is in the title of the heading for Table IV, on page 64; the title should read International Diabetes Federation (IDF) definition of metabolic syndrome - and not diabetes. The authors regret this error. The online edition of the Guideline has been corrected. 